\title{
Morphine Acutely Regulates Opioid Receptor Trafficking Selectively in Dendrites of Nucleus Accumbens Neurons
}

\author{
Helena Haberstock-Debic, ${ }^{1}$ Marc Wein, ${ }^{1,2}$ Michel Barrot,${ }^{3,6}$ Eric E. O. Colago, ${ }^{4}$ Zia Rahman, ${ }^{3}$ Rachael L. Neve, ${ }^{5}$ \\ Virginia M. Pickel, ${ }^{4}$ Eric J. Nestler, ${ }^{3}$ Mark von Zastrow, ${ }^{1}$ and Adena L. Svingos ${ }^{4}$ \\ ${ }^{1}$ Departments of Psychiatry and Cellular and Molecular Pharmacology, University of California, San Francisco, San Francisco, California 94143-2140, \\ ${ }^{2}$ Department of Psychiatry, Yale University School of Medicine, New Haven, Connecticut 06510, ${ }^{3}$ Department of Psychiatry and Center for Basic \\ Neuroscience, University of Texas Southwestern Medical Center, Dallas, Texas 75390, ${ }^{4}$ Department of Neurology and Neuroscience, Weill Medical College \\ of Cornell University, New York, New York 10021, 5 Department of Neuroscience, McLean Hospital, Belmont, Massachusetts 02178, and ${ }^{6}$ Unité Mixte de \\ Recherche, 7519 Centre National de la Recherche Scientifique/University Louis Pasteur, 67084 Strasbourg, France
}

\begin{abstract}
Morphine stimulates the internalization of $\mu$-opioid receptors (MORs) in transfected cell models to a lesser degree than opioid peptides and other analgesic drugs, such as methadone, and previous studies have reported that morphine does not produce a detectable redistribution of MORs in neural tissue after either acute or chronic administration. Nevertheless, morphine produces profound physiological effects, raising the question of whether receptor trafficking plays any role in the in vivo actions of morphine. We investigated the effects of opiate drugs on recombinant and native opioid receptors in the nucleus accumbens, which plays an important role in mediating the behavioral effects of opiate drugs. Morphine and methadone differed in their effects on the internalization of epitope-tagged MORs in cell bodies, introduced by viral gene transfer and imaged by fluorescence microscopy. A mutation of the cytoplasmic tail that confers morphine-induced internalization in cultured cells had a similar effect on receptor trafficking in nucleus accumbens cell bodies. Surprisingly, in contrast to its failure to affect MOR distribution detectably in cell bodies, acute morphine administration produced a pronounced change in MOR distribution visualized in the processes of the same neurons. A similar effect of acute morphine administration was observed for endogenously expressed MORs by immunoelectron microscopy; the acute administration of morphine increased the density of MORs associated with internal membrane structures specifically in dendrites. These results provide the first evidence that morphine regulates the distribution of MORs in neuronal processes, suggesting that "compartment-selective" membrane trafficking represents a previously unanticipated type of opioid receptor regulation contributing to the in vivo effects of opiate drugs on a physiologically relevant population of CNS neurons.
\end{abstract}

Key words: opiate; drug addiction; receptor trafficking; striatum; acute morphine administration; dendrite

\section{Introduction}

Opiate drugs such as morphine and methadone mediate their physiological effects by activating G-protein-coupled opioid receptors in CNS neurons. These drugs bind preferentially to the $\mu$ type-opioid receptor (MOR) in vitro, and gene knock-out studies indicate that the MOR is the major receptor mediating the analgesic and rewarding effects of morphine (Kieffer, 1999). Despite their similar mechanism of action, individual opiate drugs differ significantly in their effects on the cellular regulation of opioid receptors. Studies conducted primarily in non-neural cell models have established that drugs such as methadone promote the rapid endocytosis of MOR, a fundamental regulatory process also induced by opioid peptides. In contrast, morphine does not promote detectable endocytosis of MOR in cultured cells after prolonged (Arden et al., 1995) or acute (Keith et al., 1996; Whistler and von Zastrow, 1998; Zhang et al., 1998) administration. Nevertheless, it is well es-

Received Dec. 11, 2002; revised Feb. 11, 2003; accepted Feb. 11, 2003.

This work was supported by research grants from the National Institutes of Health (National Institute on Drug Abuse and National Institute of Mental Health). H.H.D. was supported by a National Research Service Award (DA06003). We thank Dr. Oscar Marin for valuable advice and discussion.

Correspondence should be addressed to Mark von Zastrow, Box 2140, N212 Genentech Hall, University of California, 600 16th Street, San Francisco, CA 94143-2140. E-mail zastrow@itsa.ucsf.edu.

Copyright $\odot 2003$ Society for Neuroscience $\quad$ 0270-6474/03/234324-09\$15.00/0 tablished that morphine produces profound physiological tolerance and dependence in vivo (Nestler, 2001). These observations have led to considerable discussion of what role, if any, cellular mechanisms of opioid receptor trafficking play in the physiological adaptation to morphine in the intact CNS.

A potential limitation of our present understanding is that it is derived, in part, from studies of cloned receptors expressed in non-neural cell lines. Therefore, a critical question is what effect morphine has on the regulation of opioid receptors in CNS neurons. Electrophysiological and biochemical evidence indicate that morphine induces functional desensitization of opioid signaling (Sim et al., 1996; Williams et al., 2001) and that morphinetolerant animals show adaptations in postreceptor signaling proteins (Nestler, 2001). However, immunocytochemical studies failed to reveal any effect of morphine on the subcellular localization of MORs in brain and spinal cord neurons, whereas certain other analgesics induce a rapid redistribution of receptors, consistent with ligand-induced endocytosis (Sternini et al., 1996; Keith et al., 1998; Trafton et al., 2000; He et al., 2002). These observations led to the proposal that physiological adaptations of the CNS to morphine may be mediated by the modification of "downstream" signaling machinery and neural circuitry (Alvarez et al., 2001; Borgland, 2001; Kieffer and Evans, 2002). 
An alternative hypothesis is that morphine does regulate the membrane trafficking of opioid receptors, but that the type of regulation involved in vivo has eluded experimental detection. Here, we used two complementary approaches to perform a detailed examination of the effects of morphine on the membrane trafficking of MORs in the rat nucleus accumbens, a brain region important for the rewarding and addictive properties of morphine and other drugs (Goeders et al., 1984; Hakan and Henriksen, 1987; West and Wise, 1988; Christie et al., 1997; Nestler, 2001). First, we have compared the effects of morphine and methadone on the localization of epitope-tagged wild-type and mutant opioid receptors overexpressed in the nucleus accumbens neurons by the injection of a viral gene transfer vector. Second, we used immunoelectron microscopy to investigate at high resolution the effects of morphine on the localization of endogenously expressed MORs in defined neuronal compartments. Our results confirm the existence of drug-specific differences in the effects of opiate drugs on the membrane trafficking of MOR in cell bodies. In addition, they reveal novel and surprising effects of morphine on opioid receptor trafficking specifically in dendrites.

\section{Materials and Methods}

Vectors and packaging of viral constructs

Packaging of the viral vectors has been described previously (Neve et al., 1997). Briefly, HindIII/XbaI fragments encoding hemagglutinin (HA) epitope-tagged wild-type MORs (Kaufman et al., 1995; Keith et al., 1996) and MOR $/ \delta$ opioid receptor (DOR) chimeric mutant opioid receptors (Whistler et al., 1999) were inserted into the herpes simplex virus (HSV) amplicon HSVPrpUC, packaged with helper 5dl1.2, purified on a sucrose gradient, pelleted, and resuspended in $10 \%$ sucrose. The average titer of the viral stocks was $2 \times 10^{7}$ infectious units per milliliter; transgene expression was regulated by the HSV IE4/5 promoter.

\section{Stereotaxic infections and animal treatment}

HSV vectors were delivered by stereotaxic microinjections into the nucleus accumbens. Male Sprague Dawley rats were anesthetized with sodium pentobarbital and placed in a Kopf stereotaxic apparatus (David Kopf Instruments, Tujunga, CA). The skull was exposed, and the tips of two Hamilton microsyringes were aligned to the bregma. Two microliters of virus were injected slowly over $10 \mathrm{~min}$ at coordinates $1.8 \mathrm{~mm}$ anterior, $2.5 \mathrm{~mm}$ lateral ( $10^{\circ}$ angle), and $6.8 \mathrm{~mm}$ ventral to the bregma. For in situ hybridization, animals received unilateral injections of the chimeric or wild-type viral construct. For immunocytochemistry, animals received bilateral injections of the chimeric construct in the left hemisphere and the wild-type construct in the right hemisphere. After injection, the needle was allowed to rest in place for an additional $5 \mathrm{~min}$ to allow time for diffusion of the viral stock. Animals were allowed to recover for 3-4 d after surgery. For in situ hybridization, animals were then killed by decapitation. The brains were rapidly dissected and frozen on dry ice, and coronal cryosections were collected and thaw-mounted onto charged slides. For immunocytochemistry, animals received intraperitoneal or subcutaneous injections of morphine $(20$ or $5 \mathrm{mg} / \mathrm{kg}$, respectively), methadone ( $4 \mathrm{mg} / \mathrm{kg}$ ), or saline; they were killed $30 \mathrm{~min}$ later by the injection of chloral hydrate and transcardial perfusion with $50 \mathrm{ml}$ ice-cold PBS followed by $400 \mathrm{ml}$ of ice-cold $4 \%$ paraformaldehyde. Dissected brains were placed in perfusate for $2 \mathrm{hr}$ and cryoprotected in $30 \%$ sucrose overnight.

\section{In situ hybridization}

Antisense riboprobes specific for either MOR or DOR tail mRNA were generated by adding a T7 promoter start site to the minus strand of PCR fragments of these respective coding regions. PCR fragments were used as templates for in vitro transcription reactions with T7 RNA polymerase and S35-UTP. Radioactive transcripts were purified by spin column gel filtration (Hoffmann-La Roche, Nutley, NJ) for hybridization. Pretreatment and hybridization procedures were performed essentially as established by Decimo et al. (1993). Briefly, slide-mounted sections were fixed in $4 \%$ paraformaldehyde, treated with glycine followed by acetic anhydride containing $0.1 \mathrm{M}$ triethanolamine, dehydrated through a graded series of alcohols, defatted with chloroform, and hybridized with S35UTP riboprobes overnight at $60^{\circ} \mathrm{C}$. After hybridization, sections were treated with RNase A, washed to a stringency of $0.1 \times$ SSC, dried, and exposed to max Hyperfilm (Amersham Biosciences, Piscataway, NJ). Films were developed after being allowed to expose for $3 \mathrm{~d}$.

\section{Brain preparations and immunocytochemical localization of epitope-tagged receptors}

Fixed brains were dissected and cryoprotected in 30\% sucrose-PBS. Cryostat sections ( $40 \mu \mathrm{m}$ thick) were rinsed in PBS and then incubated free-floating in $1 \%$ bovine serum albumin (BSA) and $0.3 \%$ Triton X-100 in PBS for $30 \mathrm{~min}$ at room temperature. Sections were incubated with monoclonal anti-HA antibody (HA.11, $5 \mu \mathrm{g} / \mathrm{ml}$; Covance, Princeton, $\mathrm{NJ}$ ) for $36 \mathrm{hr}$ at $4^{\circ} \mathrm{C}$ in $0.3 \%$ Triton $\mathrm{X}-100 / \mathrm{PBS} / 1 \% \mathrm{BSA}$, washed extensively by multiple changes of PBS at room temperature, and then incubated with goat anti-mouse antibody conjugated with the cyanine dye Cy3 (1:500; Jackson ImmunoResearch, West Grove, PA) in 0.3\% Triton $\mathrm{X}-100 / \mathrm{PBS} / 0.5 \% \mathrm{BSA}$ for $2 \mathrm{hr}$ at room temperature. Sections were again washed extensively in PBS and mounted to glass slides for fluorescence microscopy. Epifluorescence microscopy was performed using an inverted microscope fitted with a Nikon (Tokyo, Japan) PlanApo $10 \times /$ numerical aperture (NA) 0.5 or PlanApo $20 \times /$ NA 0.5 dry objectives for low-magnification viewing, and a Nikon PlanApo $60 \times /$ NA 1.4 oil objective for high-magnification viewing. Confocal fluorescence microscopy was performed using a Bio-Rad (Hercules, CA) MRC1000 laser scanning microscope fitted to an inverted microscope (Zeiss, Oberkochen, Germany) and a Zeiss PlanApo $100 \times /$ NA 1.3 objective. The number of cell bodies and processes displaying peripheral (membrane) receptor localization or punctate, intracellular localization were counted using coded slides on 30-60 cell bodies and 30-60 processes per animal (the exact number varied depending on how many structures could be unambiguously classified in each set of coded sections). Receptor localization was quantified in the nucleus accumbens on 5-7 coronal tissue sections (40 $\mu \mathrm{m}$ thick) per animal, which were taken at levels 11-14 according to the Paxinos and Watson rat brain atlas (1982). Six to eight animals were analyzed for each treatment condition.

To examine the expression of recombinant receptor protein in the nucleus accumbens, we used immunocytochemical methods and an anti-HA antibody that specifically detects the epitope-tagged receptor with very low nonspecific background staining. The examination of tissue sections by epifluorescence microscopy at low magnification revealed immunoreactive receptors in neurons located throughout the nucleus accumbens of the injected side (see Fig. 1b), as well as in a small number of neurons located immediately ventral to this region. By morphological criteria, essentially all transgene-expressing cells observed in the nucleus accumbens appeared to be neurons, consistent with the high degree of neurotropism of this virus. Consistent with the previously established ability of this viral vector to mediate transgene expression without substantial cytotoxicity (Carlezon et al., 1997, 2000), the expression of recombinant receptors was not associated with any detectable change in neuronal morphology or tyrosine hydroxylase immunoreactivity (data not shown). Results of cell counts are expressed as means \pm SD. Statistical analysis of differences between control (untreated) and experimental (drug-treated) groups was performed using a Student $t$ test.

\section{Electron microscopy}

Acute morphine administration and tissue preparation. Six adult male Sprague Dawley rats were injected intraperitoneally with saline or 20 $\mathrm{mg} / \mathrm{kg}$ morphine $30 \mathrm{~min}$ before being killed and the brain tissue being fixed ( $n=3$ control and 3 experimental). Brain tissue was processed based on the methods described previously (Chan et al., 1990). Briefly, animals were anesthetized with sodium pentobarbital $(100 \mathrm{mg} / \mathrm{kg}$, i.p.) and perfused sequentially through the ascending aorta with the following: $40 \mathrm{ml}$ heparin $(1000 \mathrm{U} / \mathrm{ml}$ in $0.15 \mathrm{M} \mathrm{NaCl}), 50 \mathrm{ml}$ acrolein $(3.8 \%$; Polysciences, Warrington, PA), and $200 \mathrm{ml}$ paraformaldehyde (2\%) in $0.1 \mathrm{~m}$ phosphate buffer ( $\mathrm{PB}, \mathrm{pH} 7.4)$. The brains were removed, cut into $4-5 \mathrm{~mm}$ coronal blocks, and postfixed for $30 \mathrm{~min}$ in $2 \%$ paraformalde- 


\section{a}

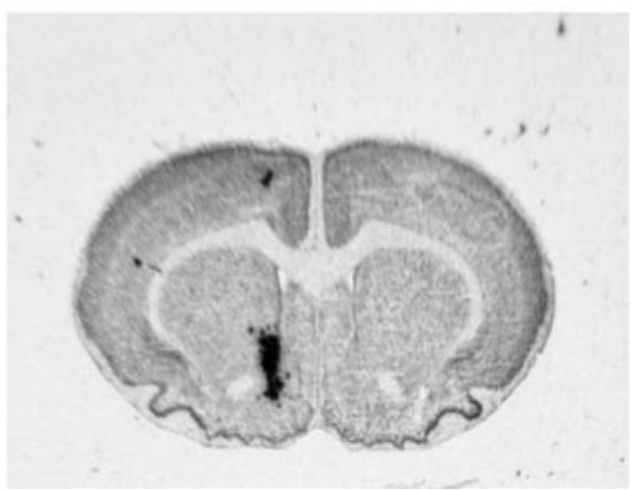

b

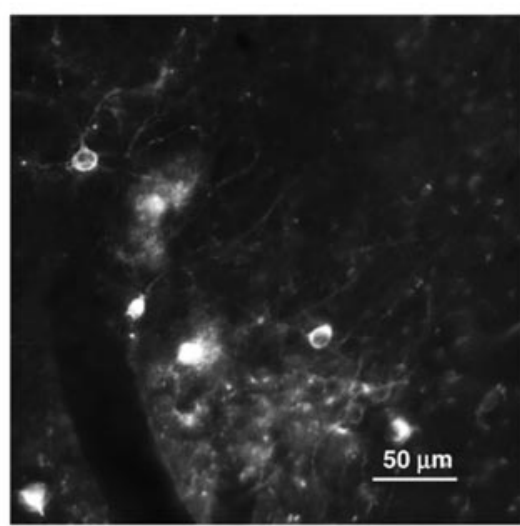

Figure 1. Expression of opioid receptors in the nucleus accumbens after viral gene transfer. $a$, In situ hybridization of a brain section prepared $4 \mathrm{~d}$ after the stereotaxic injection of $\mathrm{HSV}$ vectors encoding MOR/DOR (left) and MOR (right). Displayed is the mRNA expression signal using a riboprobe recognizing a terminal sequence in the DOR tail, which is present in the recombinant MOR/DOR mutant receptor but not in wild-type MOR (see Materials and Methods). $b$, Epifluorescence microscopy image showing HA-tagged MOR/DOR protein expression in a section through the left nucleus accumbens after HSV-mediated gene transfer (imaged using a 10× objective).

hyde. Sections through the rostrocaudal extent of the nucleus accumbens were cut on a vibratome at a thickness of $30-40 \mu \mathrm{m}$, incubated for 30 min in a solution of $1 \%$ sodium borohydride in $\mathrm{PB}$ to remove active aldehydes, and rinsed in PB. Tissue sections were then rinsed in Trisbuffered saline (TBS, pH 7.6), and incubated for $30 \mathrm{~min}$ in $1 \% \mathrm{BSA}$ in TBS to minimize nonspecific labeling.

Immunocytochemical detection. Tissue sections from control and experimental groups were coded using hole punches and processed simultaneously for immunocytochemical visualization using a well characterized rabbit polyclonal MOR antiserum (Arvidsson et al., 1995) commercially obtained from Diasorin Inc. (Stillwater, MN). For pre-embedding immunogold-silver labeling (Chan et al., 1990), tissue sections were incubated for $48 \mathrm{hr}$ at $4^{\circ} \mathrm{C}$ in a primary antibody solution that contained the MOR antiserum (1:5000) in $0.1 \% \mathrm{BSA} /$ TBS. Tissue sections were then (1) incubated for $2 \mathrm{hr}$ in colloidal gold-labeled (1 nm) goat anti-rabbit (Amersham Biosciences, Arlington, Heights, IL) IgG (1:50), (2) fixed for $10 \mathrm{~min}$ in $2 \%$ glutaraldehyde in PBS, and (3) reacted for $5 \mathrm{~min}$ with a silver solution (Amersham Biosciences).

Immunolabeled tissue sections were fixed for $60 \mathrm{~min}$ in $2 \%$ osmium tetroxide, dehydrated in a series of graded ethanols and propylene oxide, and incubated overnight in a 1:1 mixture of propylene oxide and Epon 812 (Electron Microscopy Sciences, Fort Washington, PA). The tissue was then transferred to $100 \%$ Epon for $2-3 \mathrm{hr}$ and flat-embedded between two pieces of Aclar plastic. Ultrathin sections $(40-50 \mathrm{~nm})$ through the nucleus accumbens at levels 11-13 of the Paxinos and Watson rat brain atlas (1982) were cut using a diamond knife (Diatome, Warrington, PA). To control for any differences in the penetration of gold particles within the tissue, ultrathin sections were cut on an LKB ultramicrotome (LKB-Wallac, Gaithersburg, MD) exclusively from the outer surface of the plastic-embedded tissue. The thin sections were placed onto copper grids, counterstained with lead citrate and uranyl acetate, and examined with a Phillips (Mahwah, NJ) 201 electron microscope at a magnification of $10,000 \times$.

Electron microscopic data analysis. Three vibratome sections taken from each of the rats were used for a total field analysis of $24,092 \mu \mathrm{m}^{2}$. Micrographs used for the analysis were taken from the Epon-tissue interface and were captured by an observer who was unaware of the conditions. MOR-labeled profiles were divided by neuronal subcompartment (cell bodies, dendrites, dendritic spines, and axons), as described previously (Svingos et al., 1996). A second observer, who was also unaware of the conditions, analyzed the number of immunogold-silver particles/neuronal subcompartments that were located either within the cytoplasm or in contact with the internal surface of the plasma membrane. Profiles that were considered labeled contained gold-silver parti- cles that overlaid defined structures, and numbered equivalent to at least twice the background. Statistical differences in (1) total gold particles, (2) cytoplasmic MOR, and (3) membrane-bound MOR between control and experimental animals and among neuronal subcompartments were determined using an ANOVA followed by a Neuman-Keuls post hoc analysis. Values are expressed as the density of MOR gold particles (means \pm SEM) per square micrometer.

\section{Results}

Viral-mediated gene transfer of epitope-tagged opioid receptors in nucleus accumbens neurons

As one approach to investigate opioid receptor trafficking in physiologically relevant CNS neurons in vivo, we used a previously established HSV-mediated gene transfer system for expressing recombinant proteins in the nucleus accumbens of adult rats (Neve et al., 1997). One virus encoded an HA-tagged version of the wild-type MOR1, a predominant form of MORs expressed in brain (Kaufman et al., 1995; Zimprich et al., 1995; Pan et al., 1999). A second virus encoded an HA-tagged chimeric mutant receptor (Whistler et al., 1999), in which a portion of the C-terminal cytoplasmic domain of MOR1 is replaced by the corresponding sequence from the DOR (MOR/DOR chimera). This mutation confers rapid, morphine-induced internalization of receptors in cell culture systems via a highly conserved mechanism involving G-protein-coupled receptor kinase (GRK) and arrestin-mediated association of receptors with clathrin-coated pits (Goodman et al., 1998; Whistler and von Zastrow, 1998; Zhang et al., 1998; Whistler et al., 1999). Recombinant viruses were introduced into the rat nucleus accumbens using stereotaxic injection. To examine the precision and success of the viralmediated transfer of opioid receptors in this brain region, we examined the level of mRNA expression for both receptors by in situ hybridization using riboprobes that recognize sequences encoding the distinct cytoplasmic tails of MOR and MOR/DOR mutant receptors. mRNA encoding recombinant receptor was detected specifically in the nucleus accumbens and was expressed at high levels relative to the endogenous receptor mRNA (Fig. 1a). Fluorescence microscopy using anti-HA confirmed that recombinant receptors were expressed mainly in the nucleus accumbens. Recombinant receptors were expressed specifically in neurons and were not detected in astrocytes, as indicated by a lack of colocalization with glial fibrillary acidic protein (data not 


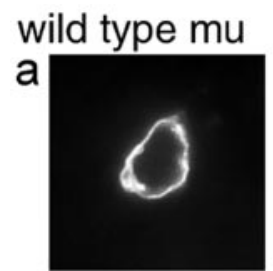

$a^{\prime}$

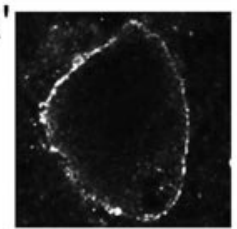

Saline

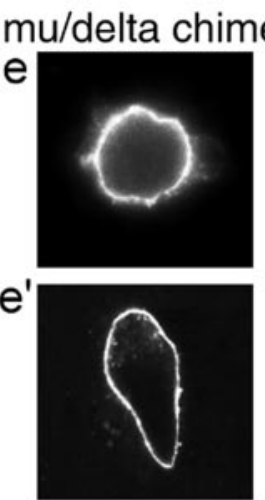

Saline
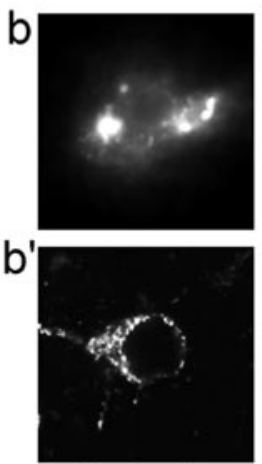

Methadone
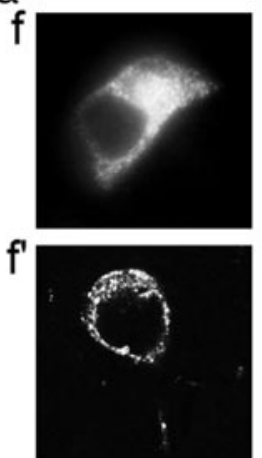

Methadone

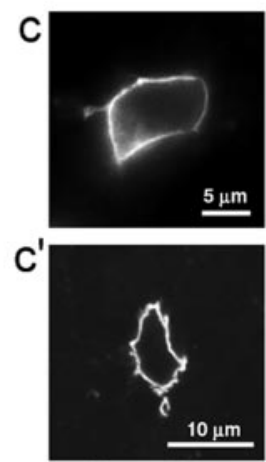

Morphine

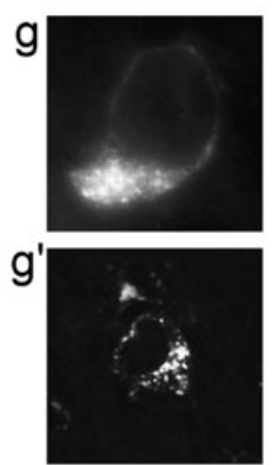

Morphine

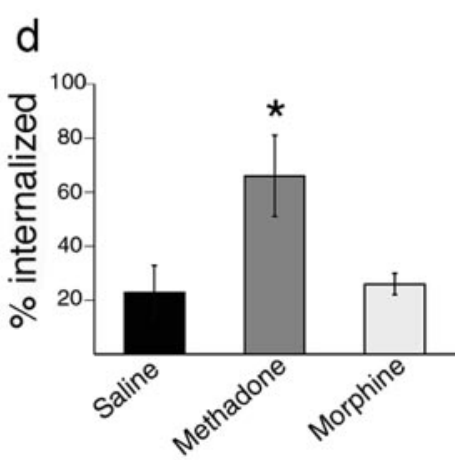

h

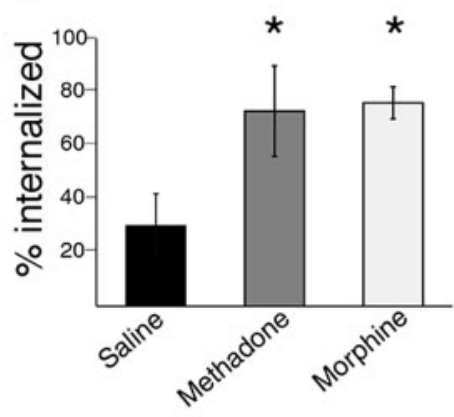

Figure 2. Effects of acute administration of methadone and morphine on wild-type MOR (wild type mu) and MOR/DOR (mu/delta chimera) in neuronal cell bodies in nucleus accumbens. $a-c$, Epifluorescence images of nucleus accumbens sections using anti-HA to visualize expressed HA-MOR (imaged using a $60 \times$ objective). Representative images from saline-injected ( $a$ ), methadoneinjected $(b)$, and morphine-injected $(c)$ animals are shown. Confocal optical sections of similar specimens are shown in $a^{\prime}-c^{\prime}$, respectively. $d$, Summary of blinded analysis of multiple sections by immunofluorescence microscopy. Bars represent the mean fraction of neuronal cell bodies visualized in which expressed MOR was localized predominantly in intracellular membrane structures (see Materials and Methods), as determined from 30-60 cell bodies per animal derived from six to eight animals per condition. Error bars indicate means \pm SD. * $p<0.05$; Student's $t$ test. $e-h$, The corresponding analysis of HA-MOR/DOR localization.

shown), consistent with previous characterization of this viral expression system (Carlezon et al., 1997, 2000).

\section{Agonist-specific differences in opioid receptor internalization observed in neuronal cell bodies}

We next performed epifluorescence and confocal fluorescence microscopy at a high magnification to examine in greater detail the localization of epitope-tagged opioid receptors in cell bodies. In saline-injected animals, HA-tagged MOR was visualized primarily at the periphery of the cell body, consistent with plasma membrane localization (Fig. 2a). This distribution of receptors was confirmed in confocal optical sections (Fig. $2 a^{\prime}$ ). The injection of methadone $(4 \mathrm{mg} / \mathrm{kg}) 30 \mathrm{~min}$ before killing the animals produced a pronounced change in the localization of epitopetagged receptors. Reduced immunoreactivity was observed in the majority of neurons at the periphery of the cell body, and receptor immunoreactivity was visualized predominantly in a punctate distribution throughout the cytoplasm (Fig. 2b). Receptorcontaining puncta present in the cell body could be resolved clearly from the plasma membrane in confocal optical sections (Fig. $2 b^{\prime}$ ); therefore, they probably represent cytoplasmic vesicles. In contrast, the injection of animals with morphine, even at a high dose $(20 \mathrm{mg} / \mathrm{kg})$, failed to induce a detectable redistribution of HA-tagged MORs. This was indicated both by epifluorescence and confocal microscopy (Fig. $2 c, c^{\prime}$ ) and was confirmed by the quantification of multiple neurons selected at random in coded specimens (Fig. $2 d$ ). In this analysis, 30-60 cell bodies were counted per animal, and tissue sections from 6-8 animals were analyzed for each treatment group.

The HA-tagged MOR/DOR mutant opioid receptor, like the tagged wild-type MOR, was localized predominantly in the plasma membrane of neuronal cell bodies in saline-treated animals (Fig. 2e,e'). Also similar to the wild-type MOR, methadone $(4 \mathrm{mg} / \mathrm{kg}$ ) produced a pronounced redistribution of somatic MOR/DOR mutant receptors to brightly stained puncta (Fig. $2 f$ ) visualized by epifluorescence microscopy. Many of these puncta could be resolved from the plasma membrane in confocal optical sections, suggesting that they represent cytoplasmic vesicles (Fig. $2 f^{\prime}$ ). In contrast to its failure to induce detectable redistribution of wild-type MOR in cell bodies, morphine induced a rapid redistribution of MOR/DOR mutant receptors to cytoplasmic vesicles. This effect was observed $30 \mathrm{~min}$ after the administration of morphine at $20 \mathrm{mg} / \mathrm{kg}$ (Fig. $2 g, g^{\prime}$ ) as well as at $5 \mathrm{mg} / \mathrm{kg}$ (data not shown). This is consistent with the ability of the MOR/DOR mutant receptor to undergo morphine-induced endocytosis in cultured cells, and was confirmed by blinded analysis of multiple specimens (Fig. $2 h$ ). Furthermore, because the experimental design involved injecting viruses encoding wild-type and mutant receptors into opposite sides of the brain by stereotaxic injection, differences in the effects of morphine on MOR and MOR/DOR trafficking were observed in the same brain sections. 


\section{wild type mu}

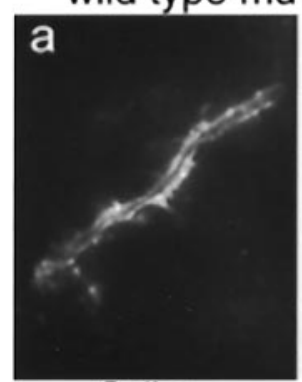

Saline

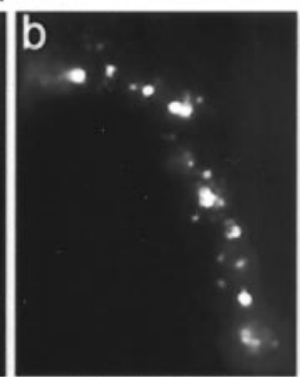

Methadone

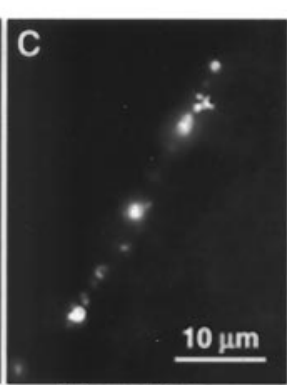

Morphine

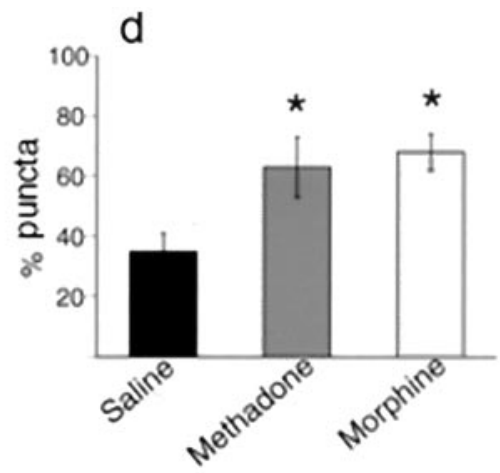

mu/delta chimera

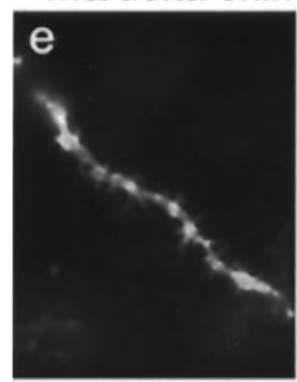

Saline

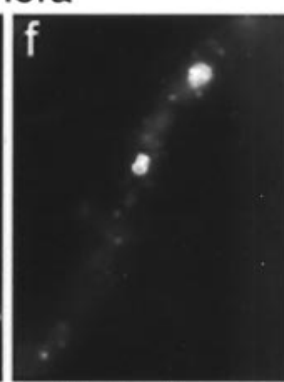

Methadone

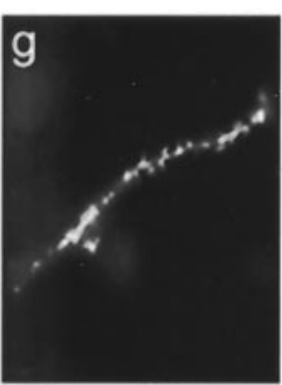

Morphine

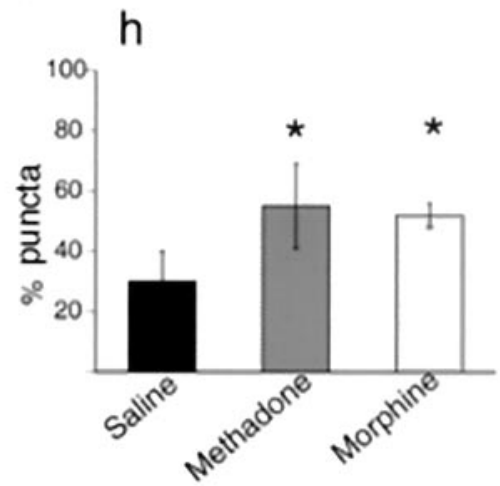

Figure 3. Effects of acute administration of methadone and morphine on wild-type MOR and MOR/DOR receptors in processes of nucleus accumbens neurons. Representative epifluorescence images ( $60 \times$ objective) are shown, illustrating the predominant pattern of MOR localization in processes from saline-injected $(a)$, methadone-injected $(b)$, or morphine-injected $(c)$ animals. $d$, Summary of blinded analysis of multiple sections. Error bars indicate the mean fraction of neuronal processes in which MOR immunoreactivity was visualized predominantly in a discontinuous or punctate distribution (see Materials and Methods), as determined from the examination of $30-60$ processes per animal, derived from six to eight animals per condition. Error bars indicate means \pm SD. ${ }^{*} p<0.05$; Student's $t$ test. $e-h$, Corresponding analysis of MOR/DOR mutant receptor visualization in processes.

\section{Both morphine and methadone affect the appearance of MOR} in neuronal processes

Morphine produced unexpected effects on the wild-type MOR visualized in the nucleus accumbens processes. In control (salineinjected) animals, HA-tagged wild-type MOR as well as MOR/ DOR mutant receptors were visualized predominantly (70\%) in processes containing receptors in a relatively smooth distribution (Fig. 3a,e). In tissue prepared from methadone-injected animals $(4 \mathrm{mg} / \mathrm{kg}$ ), there was a pronounced increase in the number of receptor-containing processes brightly stained with opioid receptors in a discontinuous pattern (Fig. $3 b, f$ ). Consistent with its ability to promote the redistribution of MOR/DOR mutant receptors in cell bodies, the majority of neuronal processes in morphine-injected animals exhibited staining of MOR/DOR mutant receptors in a punctate or discontinuous pattern (Fig. $3 g$ ). Surprisingly, morphine also induced a pronounced change in the appearance of wild-type MOR, such that the majority of processes visualized were characterized by bright receptor labeling in a discontinuous or punctate pattern (Fig. $3 c$ ). This effect was observed both in animals administered $20 \mathrm{mg} / \mathrm{kg}$ morphine (Fig. $3 c$ ) and $5 \mathrm{mg} / \mathrm{kg}$ (data not shown). The blinded analysis of multiple specimens expressing MOR or MOR/DOR mutant receptors confirmed this observation, and also indicated that morphine and methadone have similar effects on wild-type MOR in the processes of nucleus accumbens neurons (Fig. 3d,h) $(30-60$ processes counted per animal, six to eight animals analyzed per treatment).
Morphine produces different effects on opioid receptors present in the cell body relative to processes of the same neuron

Because viral vectors were injected specifically into the nucleus accumbens, transgene-expressing neurons are expected to be limited to neurons whose cell bodies are present in this structure. Although the nucleus accumbens contains predominantly ( $\sim 90 \%)$ cell bodies of GABAergic neurons, several subtypes of these cells have been identified, and a number $(\sim 10 \%)$ of cholinergic interneurons also originate in this structure (Kita and Kitai, 1988; Meredith et al., 1989). This consideration raised the question of whether morphine induces different effects on opioid receptors present in the cell body relative to processes of the same neuron, or whether the observed effects may reflect differences in the membrane-trafficking properties of opioid receptors in distinct neuronal subpopulations. To address this question we examined the effects of morphine and methadone on opioid receptor localization in the cell body and processes visualized in the same neurons. Neurons expressing recombinant receptors were identified at low magnification, then receptor localization was examined at higher magnification in distinct membrane domains of the same defined neuron.

Representative examples of this distribution are shown in Figure 4. In control (saline-injected) animals, MOR and MOR/DOR mutant receptors were visualized in a smooth peripheral distribution both in the cell body and in associated neuronal processes (Fig. 4a,b). In morphine-injected animals we observed many 

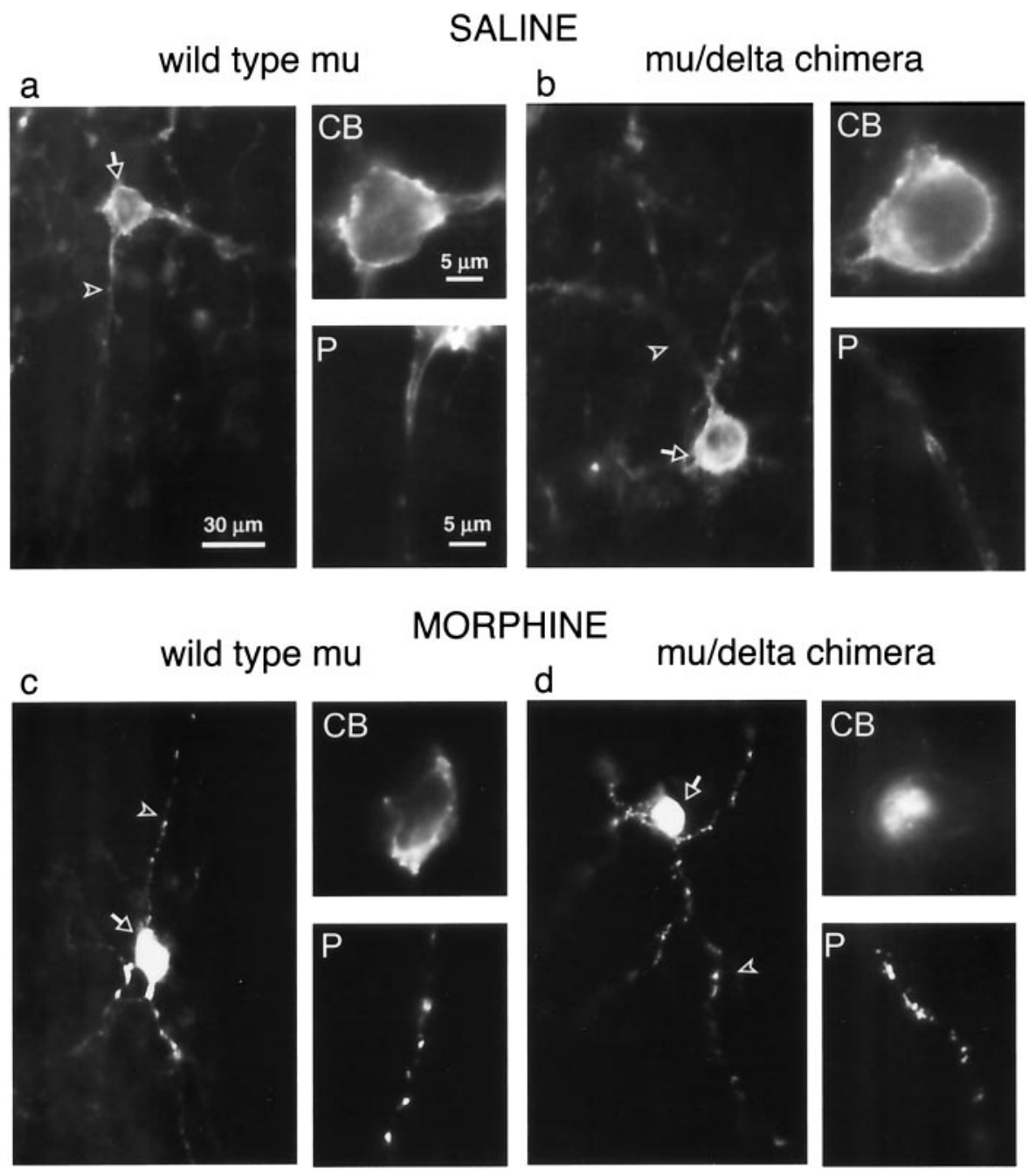

\section{$\mathrm{mu}$}

MORPHINE
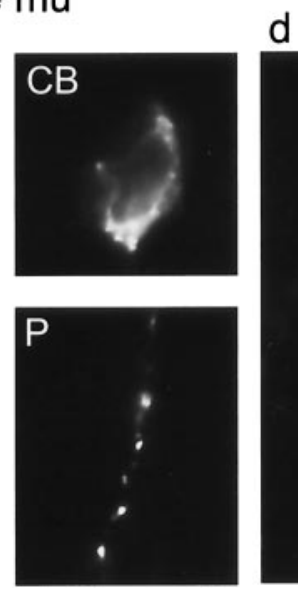

mu/delta chimera
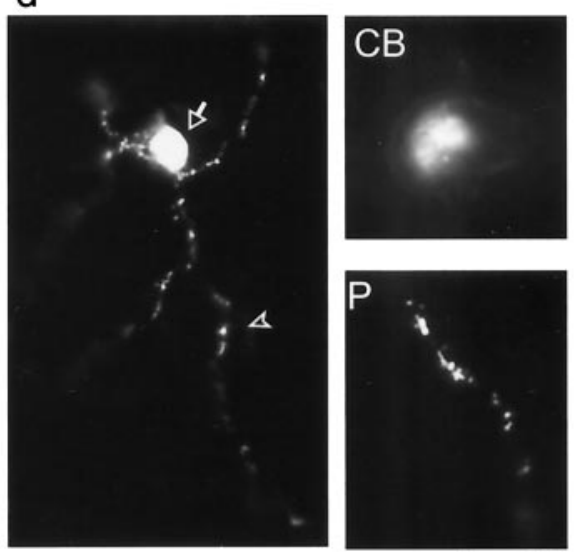

Figure 4. Effect of acute morphine administration on the distribution of wild-type MOR and MOR/DOR receptors observed in the cell body compared with associated processes of the same nucleus accumbens neuron. Representative MOR or MOR/DORexpressing neurons visualized at low magnification $(20 \times$ objective) in sections of virally infected nucleus accumbens are displayed. Insets display images of the cell body (CB) or a representative portion of associated processes (P) from the same identified neuron imaged at higher magnification ( $60 \times$ objective). $a, b$, Localization of MOR or MOR/DOR mutant receptors in salineinjected animals. c, d, Localization of MOR or MOR/DOR mutant receptors in morphine-injected animals.

internal membranes in morphologically defined neuronal compartments (i.e., cell bodies, dendrites, dendritic spines, axons) (Chan et al., 1990); we took advantage of the established ability of this method to detect endogenously expressed MOR in specimens prepared from uninfected animals (Svingos et al., 1996). As expected, the highest concentration of MOR immunoreactivity was observed in postsynaptic structures, including dendrites and cell bodies, although detectable labeling of axon terminals was also observed. Also consistent with our previous studies, the vast majority of MOR immunoreactivity was associated with the surface membrane in all neuronal compartments. In particular, very little immunoreactive MOR was detected in the internal membranes of dendrites (Svingos et al., 1996).

In animals killed $30 \mathrm{~min}$ after a single injection of morphine $(20 \mathrm{mg} / \mathrm{kg})$, a pronounced increase in the amount of endogenous MOR immunoreactivity was detected in the internal membranes of dendrites. As illustrated in a representative electron micrograph derived from a morphine-injected animal (Fig. 5), MOR immunoreactivity (arrowheads) was associated with both the surface membrane and internal membranes of dendrites, in contrast with the almost exclusive surface localization observed in untreated animals (data not shown) (Svingos et al., 1996). Blinded analysis of multiple sections confirmed this impression and indicated that this effect was highly specific for MORs present in dendrites, because there was no detectable increase in the amount of intracellular MORs visualized in the same sections in

neurons in which MOR remained in a smooth, peripheral staining pattern in the cell body yet appeared in a punctate or discontinuous pattern in associated processes (Fig. 4c). As expected, MOR/DOR mutant receptors were visualized in a punctate pattern in both the cell body and associated processes after acute morphine injection (Fig. $4 d$ ). Furthermore, methadone changed the appearance of both MOR and MOR/DOR mutant receptors visualized in both the cell body and associated processes (data not shown). Thus, morphine selectively affects the localization of MOR in processes relative to the cell body of the same neuron.

\section{Morphine acutely increases the internal membrane pool of endogenous MOR specifically in dendrites}

The studies of virally infected brain tissue were restricted to expressed (epitope-tagged) opioid receptors, and it was not possible using epifluorescence or confocal microscopy to resolve the MOR present in internal membranes in processes because these processes are not much thicker than the resolution limits of our optical microscopy systems. Therefore, we used immunoelectron microscopy to distinguish MORs associated with surface versus cell bodies or axons (Fig. 6a). Despite the substantial and statistically significant increase in the density of immunoreactive MORs associated with internal membranes of dendrites in morphine-treated animals, we did not detect any statistically significant change in the density of plasma membrane-associated MORs in this compartment (Fig. 6b). Consistent with this, acute morphine administration produced a significant increase in the total density of MOR immunoreactivity detected specifically in the dendritic compartment, whereas no detectable change in the density of MOR labeling in axons or cell bodies was observed (Fig. 6c).

\section{Discussion}

In this study we used viral-mediated gene transfer and epitope tagging to examine the effects of acute opiate administration on the localization of wild-type and mutant opioid receptors in physiologically relevant nucleus accumbens neurons in vivo. Under the conditions used, recombinant receptors were expressed in widely separated neurons, allowing us to resolve individual receptor-expressing cell bodies and associated processes by light 


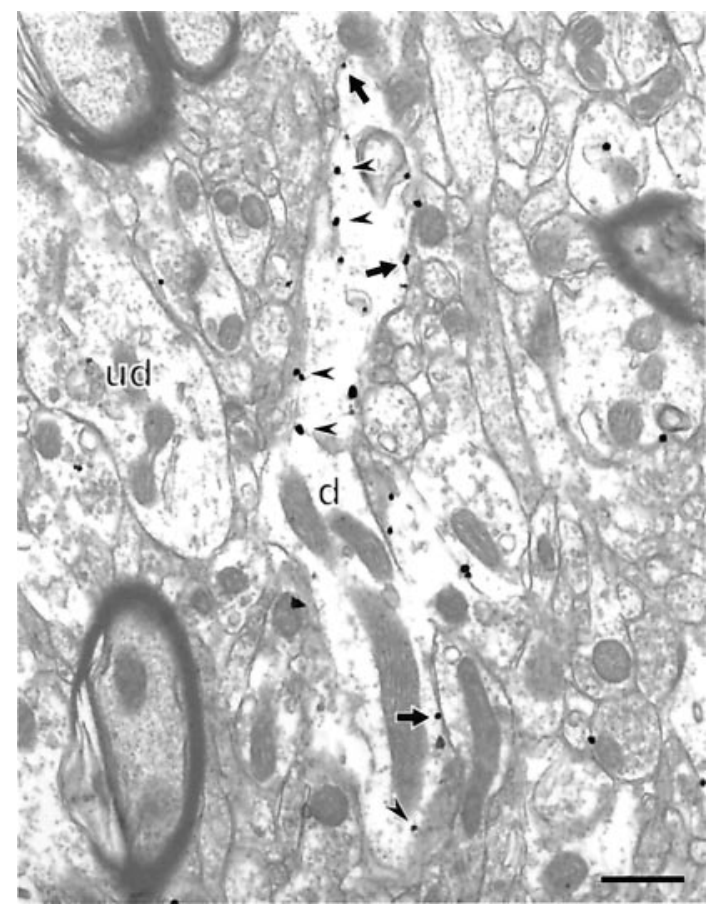

Figure 5. Ultrastructural localization of MOR immunoreactivity in nucleus accumbens dendrites after the acute administration of morphine. A representative ultrathin section showing a longitudinally cut dendritic profile from a morphine-treated ( $20 \mathrm{mg} / \mathrm{kg}, 30 \mathrm{~min}$ exposure) rat containing MOR-immunoreactive gold particles localized both to the plasma membrane (arrows) and to heterogeneous membrane structures located in the dendritic cytoplasm (arrowheads). $d$, Dendrite; ud, unlabeled dendrite. Scale bar, $0.5 \mu \mathrm{m}$.

microscopy. We also used immunoelectron microscopy to examine at higher resolution the effects of morphine on the density and distribution of endogenously expressed MOR within specific subcellular compartments in the same population of neurons.

Our studies of receptor localization in cell bodies support previous observations (namely, that acute morphine administration fails to induce detectable internalization in MOR in this compartment; Sternini et al., 1996; Keith et al., 1998; Trafton et al., 2000; He et al., 2002). The present results also indicate that the agonist selectivity of receptor internalization observed in cell bodies is modulated by the C-terminal cytoplasmic tail of the receptor, thereby providing the first in vivo evidence supporting mechanistic studies of opioid receptor trafficking conducted in cultured cells (Whistler et al., 1999). Surprisingly, in contrast to its lack of effect on MOR distribution in cell bodies, acute morphine administration induced a rapid and pronounced effect on the appearance of epitope-tagged wild-type MOR specifically in neuronal processes visualized by fluorescence microscopy. Consistent with this, acute morphine administration also affected the localization of endogenous MORs in nucleus accumbens neurons detected by immunoelectron microscopy, but did so selectively in morphologically defined dendrites without any detectable change in receptor distribution in other membrane compartments. To our knowledge, these results are the first to indicate that morphine acutely regulates intracellular opioid receptors present in a physiologically relevant population of neurons in vivo, and they also suggest that opiate drugs can have different effects on the trafficking of opioid receptors present in distinct membrane compartments of the same neuron. The effects of morphine and methadone on the localization of wild-type MOR in cell bodies is consistent with previous studies of opioid
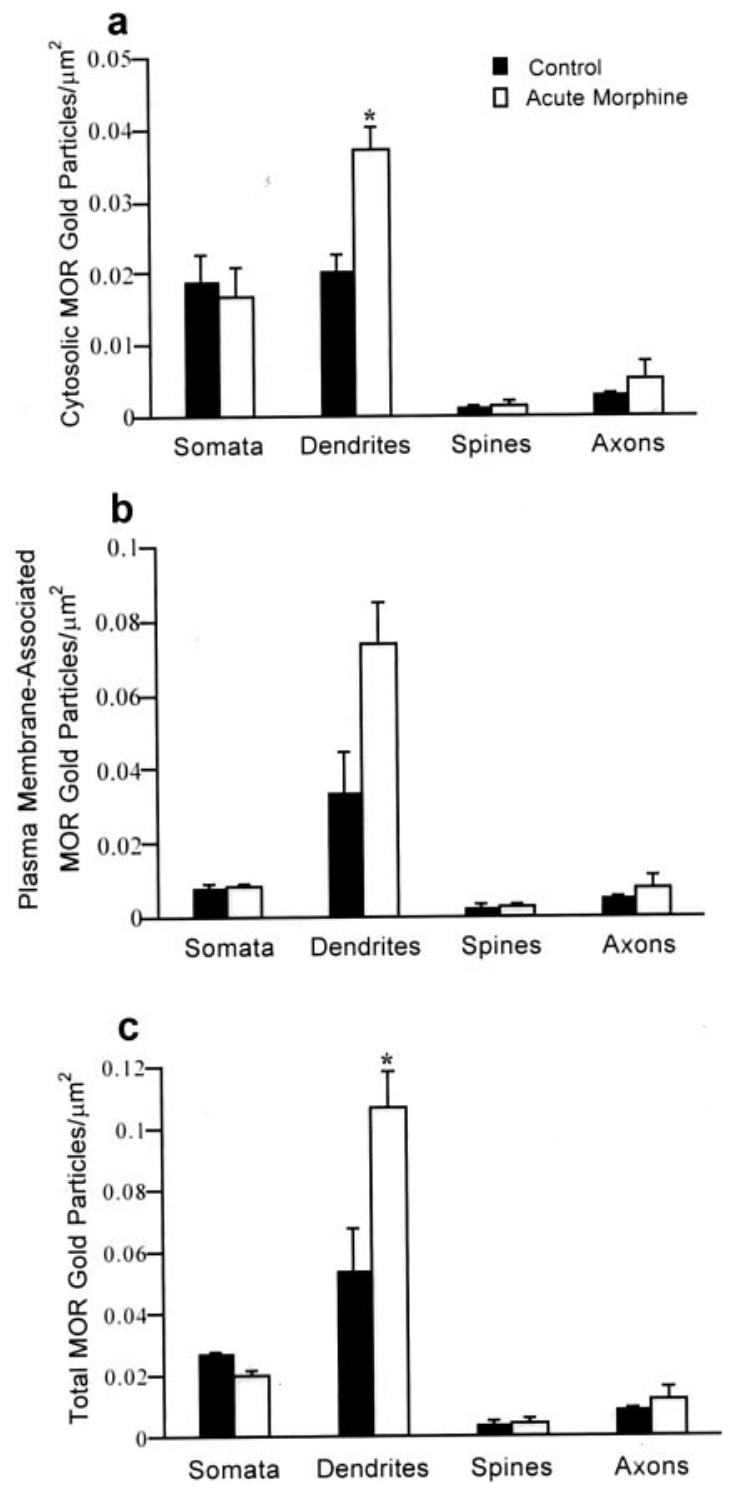

Figure 6. Acute morphine exposure increases the overall and cytoplasmic densities of MOR immunoreactivity selectively in nucleus accumbens dendrites. Area density analyses were performed to quantify the amount and location (internal vs surface membrane) of MOR gold particles in response to acute morphine administration among neuronal compartments. $a, c$, The total immunoreactive MOR gold particles (surface + internal) and mean density of MOR immunoreactivity present in internal membranes of morphologically defined dendrites were significantly enhanced after acute morphine exposure. $b$, The mean density of MORimmunoreactive gold particles associated with the surface membranes of morphologically defined neuronal compartments was not significantly altered after acute morphine administration. Values are expressed as means \pm SEM. Asterisks indicate statistically significant differences $(p<0.05)$ between groups (control vs experimental) and among neuronal compartments.

receptor endocytosis conducted in cell culture, in which endocytosis is promoted by GRK-mediated phosphorylation and the binding of arrestins to phosphorylated receptors (Whistler and von Zastrow, 1998; Zhang et al., 1998). Consistent with this, the MOR/DOR mutant receptor, which exhibits enhanced morphine-induced phosphorylation and internalization in cell culture (Whistler et al., 1999), was observed to undergo rapid internalization in nucleus accumbens cell bodies in vivo. Thus, the observed differences in the effect of acute morphine administration on MORs in dendrites suggest that there may exist functionally significant differences in the amount or activity of spe- 
cific receptor regulatory proteins, such as GRKs or arrestins, among neuronal compartments.

The punctate pattern of epitope-tagged receptor immunoreactivity observed in neuronal processes by fluorescence microscopy and the morphine-induced increase in the internal membrane pool of MOR detected by immunoelectron microscopy suggest that acute morphine administration produces rapid endocytosis of MOR selectively in dendrites. A caveat to this interpretation is that we were unable to detect a statistically significant change in the surface MOR density in dendrites in tissue sections prepared from morphine-injected animals. This is not consistent with acute morphine administration producing simply rapid internalization of MOR (which would predict a morphine-induced decrease in surface MOR). Thus, we cannot exclude the possibility that acute morphine administration, in addition to promoting the rapid internalization of MOR in dendrites, might have one or more other effects distinct from endocytosis (such as changing epitope accessibility, producing lateral redistribution of receptors, or perhaps inducing rapid biosynthesis of receptors in dendrites). However, in our studies using expressed receptors, in which the epitope used is present on the extracellular surface of the receptor protein domain and comprises an engineered sequence not likely to interact with endogenous neural machinery, strongly suggest that the acute effect of morphine on dendritic MOR is not the result of differential epitope accessibility in untreated versus morphine-injected animals. It will be interesting in future studies to investigate these other possibilities.

We believe that the present observations may be important in developing a more complete understanding of the physiological actions of opiate drugs such as morphine. It is generally believed that the acute effects of morphine do not involve receptor membrane trafficking, whereas the physiological adaptations observed after prolonged morphine administration may involve the regulation of receptors as well as downstream signaling pathways and circuitry (Nestler, 2001; Williams et al., 2001). Support for this distinction comes from previous results indicating that morphine, a prototypic opiate analgesic that produces profound acute and chronic physiological effects, fails to induce detectable redistribution of opioid receptors even when administered at high doses in vivo (Sternini et al., 1996; Keith et al., 1998; Trafton et al., 2000; He et al., 2002). The present observations strongly suggest that morphine does indeed modulate the membrane trafficking of MORs, and does so within minutes after acute administration to previously drug-naive animals. Thus, opioid receptor membrane trafficking may contribute to the acute actions of opiates and/or may represent an early event in the physiological adaptation of the nervous system to opiates. The observation that this effect of morphine is restricted to a specific dendritic membrane domain also suggests that opioid receptors present in distinct subcellular membrane compartments are differentially regulated by opiate drugs.

The mechanism distinguishing the trafficking properties of MOR in dendrites relative to other neuronal compartments remains to be elucidated. One possibility is that there exist intrinsic differences in the trafficking properties of MOR in dendrites, as might be mediated by the segregation of cytoplasmic regulatory factors in dendrites relative to other neuronal compartments. An important caveat is that the present studies were conducted on intact brain tissue using systemic opiate administration in vivo. Therefore, we cannot at present exclude the alternative possibility that the compartment-selective effects observed could be a consequence of drug-regulated changes in neural circuitry, as might be expected if morphine induces the release of another mediator of MOR trafficking (such as an endogenous opioid peptide) locally onto dendrites. This possibility might be of particular interest to addiction biology because nucleus accumbens neurons modulate the rewarding effects of opiates as well as other addictive drugs that do not bind to opioid receptors (Chefer and Shippenberg, 2002; Robinson and Berridge, 2002).

Additional studies will be necessary to address these mechanistic questions, to determine whether compartment-selective trafficking of receptors occurs in other brain regions, and to investigate the physiological consequences of the compartmentselective trafficking of opioid receptors in the acute and chronic effects of opiate drugs. The present study, by identifying a previously unanticipated type of compartment-selective opioid receptor regulation induced by morphine in a physiologically relevant population of neurons, suggests that opioid receptor membrane trafficking may play a highly specific role in mediating the acute effects of morphine in the CNS.

\section{References}

Alvarez V, Arttamangkul S, Williams JT (2001) A RAVE about opioid withdrawal. Neuron 32:761-763.

Arden JR, Segredo V, Wang Z, Lameh J, Sadee W (1995) Phosphorylation and agonist-specific intracellular trafficking of an epitope-tagged $\mu$-opioid receptor expressed in HEK 293 cells. J Neurochem 65:1636-1645.

Arvidsson U, Riedl M, Chakrabarti S, Lee JH, Nakano AH, Dado RJ, Loh HH, Law PY, Wessendorf MW, Elde R (1995) Distribution and targeting of a $\mu$-opioid receptor (MOR1) in brain and spinal cord. J Neurosci 15:3328-3341.

Borgland SL (2001) Acute opioid receptor desensitization and tolerance: is there a link? Clin Exp Pharmacol Physiol 28:147-154.

Carlezon Jr WA, Boundy VA, Haile CN, Lane SB, Kalb RG, Neve RL, Nestler EJ (1997) Sensitization to morphine induced by viral-mediated gene transfer. Science 277:812-814.

Carlezon Jr WA, Nestler EJ, Neve RL (2000) Herpes simplex virus-mediated gene transfer as a tool for neuropsychiatric research. Crit Rev Neurobiol 14:47-67.

Chan J, Aoki C, Pickel VM (1990) Optimization of differential immunogold-silver and peroxidase labeling with maintenance of ultrastructure in brain sections before plastic embedding. J Neurosci Methods 33:113-127.

Chefer VI, Shippenberg TS (2002) Changes in basal and cocaine-evoked extracellular dopamine uptake and release in the rat nucleus accumbens during early abstinence from cocaine: quantitative determination under transient conditions. Neuroscience 112:907-919.

Christie MJ, Williams JT, Osborne PB, Bellchambers CE (1997) Where is the locus in opioid withdrawal? Trends Pharmacol Sci 18:134-140.

Decimo D, Boespflug O, Meunier-Rotival M, Hadchouel M, Tardieu M (1993) Genetic restriction of murine hepatitis virus type 3 expression in liver and brain: comparative study in BALB/c and $\mathrm{C} 3 \mathrm{H}$ mice by immunochemistry and hybridization in situ. Arch Virol 130:269-277.

Goeders NE, Lane JD, Smith JE (1984) Self-administration of methionine enkephalin into the nucleus accumbens. Pharmacol Biochem Behav 20:451-455.

Goodman Jr OB, Krupnick JG, Santini F, Gurevich Santini, Penn RB, Gagnon AW, Keen JH, Benovic JL (1998) Role of arrestins in G-protein-coupled receptor endocytosis. Adv Pharmacol 42:429-433.

Hakan RL, Henriksen SJ (1987) Systemic opiate administration has heterogeneous effects on activity recorded from nucleus accumbens neurons in vivo. Neurosci Lett 83:307-312.

He L, Fong J, von Zastrow M, Whistler JL (2002) Regulation of opioid receptor trafficking and morphine tolerance by receptor oligomerization. Cell 108:271-282.

Kaufman DL, Keith Jr DE, Anton B, Tian J, Magendzo K, Newman D, Tran TH, Lee DS, Wen C, Xia YR, Lusis AJ, Evans CJ (1995) Characterization of the murine $\mu$ opioid receptor gene. J Biol Chem 270:15877-15883.

Keith DE, Anton B, Murray SR, Zaki PA, Chu PC, Lissin DV, MonteilletAgius G, Stewart PL, Evans CJ, von Zastrow M (1998) $\mu$-Opioid receptor internalization: opiate drugs have differential effects on a conserved endocytic mechanism in vitro and in the mammalian brain. Mol Pharmacol 53:377-384. 
Keith DE, Murray SR, Zaki PA, Chu PC, Lissin DV, Kang L, Evans CJ, von Zastrow M (1996) Morphine activates opioid receptors without causing their rapid internalization. J Biol Chem 271:19021-19024.

Kieffer BL (1999) Opioids: first lessons from knockout mice. Trends Pharmacol Sci 20:19-26.

Kieffer BL, Evans CJ (2002) Opioid tolerance-in search of the holy grail. Cell 108:587-590.

Kita H, Kitai ST (1988) Glutamate decarboxylase immunoreactive neurons in rat neostriatum: their morphological types and populations. Brain Res 447:346-352.

Meredith GE, Blank B, Groenewegen HJ (1989) The distribution and compartmental organization of the cholinergic neurons in nucleus accumbens of the rat. Neuroscience 31:327-345.

Nestler EJ (2001) Molecular basis of long-term plasticity underlying addiction. Nat Rev Neurosci 2:119-128.

Neve RL, Howe JR, Hong S, Kalb RG (1997) Introduction of the glutamate receptor subunit 1 into motor neurons in vitro and in vivo using a recombinant herpes simplex virus. Neuroscience 79:435-447.

Pan YX, Xu J, Bolan E, Abbadie Bolan, Chang A, Zuckerman A, Rossi G, Pasternak GW (1999) Identification and characterization of three new alternatively spliced $\mu$-opioid receptor isoforms. Mol Pharmacol 56:396-403.

Paxinos G, Watson C (1982) The rat brain in stereotaxic coordinates. New York: Academic.

Robinson TE, Berridge KC (2002) Addiction. Annu Rev Psychol 14:14.

Sim LJ, Selley DE, Dworkin SI, Childers SR (1996) Effects of chronic morphine administration on $\mu$ opioid receptor-stimulated ${ }^{355} \mathrm{GTP} \gamma \mathrm{S}$ autoradiography in rat brain. J Neurosci 16:2684-2692.

Sternini C, Spann M, Anton B, Keith Anton, Bunnett NW, von Zastrow M,
Evans C, Brecha NC (1996) Agonist-selective endocytosis of $\mu$ opioid receptor by neurons in vivo. Proc Natl Acad Sci USA 93:9241-9246.

Svingos AL, Moriwaki A, Wang JB, Uhl GR, Pickel VM (1996) Ultrastructural immunocytochemical localization of $\mu$-opioid receptors in rat nucleus accumbens: extrasynaptic plasmalemmal distribution and association with Leu5-enkephalin. J Neurosci 16:4162-4173.

Trafton JA, Abbadie C, Marek K, Basbaum AI (2000) Postsynaptic signaling via the $\mu$-opioid receptor: responses of dorsal horn neurons to exogenous opioids and noxious stimulation. J Neurosci 20:8578-8584

West TE, Wise RA (1988) Effects of naltrexone on nucleus accumbens, lateral hypothalamic and ventral tegmental self-stimulation rate-frequency functions. Brain Res 462:126-133.

Whistler JL, Chuang HH, Chu P, Jan LY, von Zastrow M (1999) Functional dissociation of $\mu$ opioid receptor signaling and endocytosis: implications for the biology of opiate tolerance and addiction. Neuron 23:737-746.

Whistler JL, von Zastrow M (1998) Morphine-activated opioid receptors elude desensitization by $\beta$-arrestin. Proc Natl Acad Sci USA 95:9914-9919.

Williams JT, Christie MJ, Manzoni O (2001) Cellular and synaptic adaptations mediating opioid dependence. Physiol Rev 81:299-343.

Zhang J, Ferguson SS, Barak LS, Bodduluri SR, Laporte SA, Law PY, Caron MG (1998) Role for G protein-coupled receptor kinase in agonistspecific regulation of mu-opioid receptor responsiveness. Proc Natl Acad Sci USA 95:7157-7162.

Zimprich A, Simon T, Hollt V (1995) Cloning and expression of an isoform of the rat $\mu$ opioid receptor (rMOR1B) which differs in agonist induced desensitization from rMOR1. FEBS Lett 359:142-146. 